\section{SP1-31 PLASMA FIBRINOGEN LEVEL AND MYOCARDIAL INFARCTION IN THE RURAL JAPANESE POPULATION: THE JMS COHORT STUDY}

doi:10.1136/jech.2011.142976n.8

${ }^{1} \mathrm{~T}$ Toyotsuji,* ${ }^{1} \mathrm{~S}$ Ishikawa, ${ }^{2} \mathrm{~K}$ Kayaba, ${ }^{1} \mathrm{Y}$ Nakamura, ${ }^{1} \mathrm{E}$ Kajii. ${ }^{1}$ Jichi Medical University, Tochigi, Japan; ${ }^{2}$ Saitama Prefectural University, Saitama, Japan

Background Plasma fibrinogen, which is an inflammation marker, is known as a risk factor for myocardial infarction among Caucasians. However there is only one study examined among Japanese, which recruited from urban area. We examined the relation between plasma fibrinogen level and myocardial infarction by using data of JMS cohort that the participants were rural Japanese.

Methods Study subjects were 6899 participants (2645 men and 4254 women) who were free of myocardial infarction, and fibrinogen level was measured in the JMS cohort study. The samples were taken between 1992 and 1995 in 12 rural areas in Japan. Cox's proportional hazard model was used to calculate the HR of myocardial infarction.

Results The average period of follow-up was $10.7 \pm 2.3$ years. Mean age was $54.7 \pm 13.4$ years in men and $55.0 \pm 12.5$ years in women. Plasma fibrinogen concentration and body mass index were significantly higher in women. The RR of myocardial infarction in the higher tertile of fibrinogen concentration was significantly higher than that in the lower tertile, after adjustment for age in men (lower vs higher tertile; HR 2.6), but not significant association was seen in women. However, even in men after adjustment for age, systolic blood pressure and smoking, the association was not significantly (lower vs higher tertile; HR 2.5).

Conclusion Our data suggest that in Japanese rural residents, high fibrinogen concentration elevated risk of myocardial infarction men, but that there is no tendency between plasma fibrinogen level and myocardial infarction in women.

\section{SP1-32 THE EPIDEMIOLOGIC STUDY OF THE PREVALENCE AND ASSOCIATED FACTORS OF METABOLIC SYNDROME AMONG HOSPITAL STAFF}

doi:10.1136/jech.2011.142976n.9

${ }^{1} \mathrm{H}$ C Shih, ${ }^{2}{ }^{2} \mathrm{~J}$ Chao, ${ }^{2,3} \mathrm{~J}$ C Luo, ${ }^{4} \mathrm{M}$ W Kuo, ${ }^{4} \mathrm{~F}$ L Chen, ${ }^{4} \mathrm{C} J$ Wei, ${ }^{5} \mathrm{~K}$ C Chen, 4,5 T H Tung. 'Department of Nursing, Kaohsiung Armed Forces General Hospital, Kaohsiung, Taiwan; ${ }^{2}$ Department of Family Medicine, Chang-Gang Memorial Hospital, Taipei, Taiwan; ${ }^{3}$ Department of Occupational Medicine, Chang-Gang Memorial Hospital, Taipei, Taiwan; ${ }^{4}$ Faculty of Public Health, School of Medicine, Fu-Jen Catholic University, Taipei, Taiwan; ${ }^{5}$ Department of Medical Research and Education, Cheng Hsin General Hospital, Taipei, Taiwan

Introduction Metabolic syndrome is a cluster of cardiovascular risk factors. From the viewpoint of preventive medicine, it is essential for early diagnosis and appropriate treatment to subjects with metabolic syndrome. The objective in this study is to explore the prevalence and associated risk factors of metabolic syndrome among hospital staff based on the health examination.

Methods Based on the cross-sectional study design, the database of the health examination of the workers in a medical center on the northern Taiwan is analysed. The definition of metabolic syndrome is according to the criteria proposed by Department of Health in 2007, Taiwan. Due to the limitation of the examination, the total cholesterol is used to replace the HDL-C value.

Results The prevalence of metabolic syndrome is $16.3 \%$ (24.8\% in male and $11.7 \%$ in female) among 1203 screened subjects. The highest proportion of abnormal index of metabolic syndrome is central obesity combined with higher blood pressure and total cholesterol. Based on the multinomial logistic regression, the significant factors related to 1-2 abnormal index of metabolic syndrome include gender (male vs female, OR 1.85, 95\% CI 1.31 to 1.47), age (OR 1.04, 95\% CI 1.02 to 1.06$)$ and obesity $(\mathrm{OR}=1.39$, $95 \%$ CI 1.31 to 1.47 ). In addition, the significant factors related to 3-5 abnormal index of metabolic syndrome also include gender (male vs female, OR 2.08, 95\% CI 1.28 to 3.45 ), age (OR 1.12, 95\% CI 1.09 to 1.14 ) and obesity (OR $1.73,95 \%$ CI 1.60 to 1.87 ).

Conclusion To promote the health promoting hospital, the priority of metabolic syndrome prevention is controlled waist circumference and body weight.

\section{SP1-33 CHANGES IN HEALTH RELATED QUALITY OF LIFE AFTER HIP OR WRIST FRACTURES DUE TO FALLS IN ELDERLY PEOPLE}

doi:10.1136/jech.2011.142976n.10

1J M Quintana, ${ }^{1} \mathrm{C}$ Las Hayas, ${ }^{2} \mathrm{~A}$ Bilbao, ${ }^{1} \mathrm{~S}$ Garcia, ${ }^{1} \mathrm{M}$ Orive, ${ }^{1} \mathrm{~N}$ Gonzalez, ${ }^{3} \mathrm{G}$ Navarro. ${ }^{1}$ Hospital Galdakao-Usansolo, Galdakao, Bizkaia, Spain; ${ }^{2}$ Bioef, Sondika, Bizkaia, Spain; ${ }^{3}$ Corporació Sanitaria Parc Taulli, Sabadell, Barcelona, Spain

Introduction Falls with fractures in elderly people are common situations. Wrist and hip fractures are the most common or severe respectively. The goal of this study was to determine the Health Related Quality of Life (HROoL) evolution, and which clinical and social factors predict its change after those fractures, by measuring HRQoL by the SF-12 questionnaire.

Methods Patients older than 65 years who attended the emergency room (ER) of seven acute hospitals with a hip or wrist fracture due to a fortuity fall were recruited. Patients fulfilled the SF-12 questionnaire at the time of the fall, as how they were before the fall, and 6 months later, as well as some other questions on sociodemographic issues. Clinical parameters from the ER and admission to the hospital were also recorded. Univariate and multivariate regression analysis were performed, considering the changes on the physical component score (PCS) domain of the SF-12 as dependent variable.

Results Analysis of our data with a recruitment of 343 hip and 412 wrist fracture patients showed an important decline in PCS (hip:10.8; wrist:8.9) at 6 months after the fracture. Older patients and lower socioeconomic status were those with greater worsening on PCS on hip fracture, after adjusting by baseline scores. On wrist fractures, older patients, women and not having social support were predictors of greater worsening.

Conclusion HRQoL physical changes in hip fractures were not influenced by socio-sanitary services while in wrist fractures social support provided benefit while it was found gender differences.

\section{SP1-34 PUBERTAL MUSCLE MASS AND DIABETES MARKERS IN CHINESE ADOLESCENTS: EVIDENCE FROM THE HONG KONG'S “CHILDREN OF 1997" BIRTH COHORT}

doi:10.1136/jech.2011.142976n.11

${ }^{1} \mathrm{~L}$ L Shi, ${ }^{*}$ S L Lee, ${ }^{1} \mathrm{~L}$ L Hui, ${ }^{1} \mathrm{~S}$ L Au Yeung, ${ }^{3} \mathrm{M}$ A Tse, ${ }^{1} \mathrm{G}$ M Leung, ${ }^{1} \mathrm{C}$ M Schooling. ${ }^{1}$ The University of Hong Kong, Hong Kong, China; ${ }^{2}$ Queen Mary Hospital, Hong Kong, China; ${ }^{3}$ Institute of Human Performance, Hong Kong, China

Introduction There is an emerging epidemic of diabetes in China despite a relatively non-obese population. Genetics and lifestyle undoubtedly play a key role. We hypothesise that environmentally driven muscle mass acquisition at puberty may also be relevant. To test this hypothesis we examined the association of sex-steroids with muscle mass and of muscle mass with fasting glucose and insulin in a sample of Chinese adolescents.

Methods In 40 adolescents (20 boys, 20 girls, age $12.9 \pm 0.1$ years), from Hong Kong's "Children of 1997" birth cohort, we assessed 